(C2007 IEEE. Personal use of this material is permitted. However, permission to reprint/republish this material for advertising or promotional purposes or for creating new collective works for resale or redistribution to servers or lists, or to reuse any copyrighted component of this work in other works must be obtained from the IEEE. 


\title{
Unbalance Power Flow Calculation for a Radial Distribution System Using Forward-Backward Propagation Algorithm
}

\author{
A. Ulinuha, M.A.S. Masoum, Senior Member, IEEE, and S.M. Islam, Senior Member, IEEE
}

\begin{abstract}
Three-phase power flow for unbalance distribution system is carried out using Forward-Backward Propagation Technique. The equivalent injection current method is employed to represent the loads and shunt admittances. The algorithm starts with mapping the distribution network to determine the forward and backward propagation paths. The backward propagation is used to calculate branch currents using the bus injection currents. The forward propagation is employed to calculate bus voltages using the obtained branch currents and line impedances. The algorithm offers robust and good convergence characteristics for radial distribution system. The algorithm is presented for the IEEE 34-bus system.
\end{abstract}

Index Terms-Convergence, forward-backward propagation, equivalent injection current, unbalance system

\section{INTRODUCTION}

$\mathrm{P}$ OWER flow calculation is backbone of power system analysis and design. It is essential for analysis of any power system in the operational as well as planning stages. The calculation is initially carried out by formulating the network equation. Node-voltage method, which is the most suitable form for many power system analyses, is commonly used. Mathematically, power flow problem requires solution of simultaneous nonlinear equations and normally employs an iterative method, such as Gauss-Seidel and Newton-Raphson.

Power flow calculation is normally performed by simply considering that the system under study is balance. Hence, the calculations are carried out for single phase assuming that the other two phases are exactly the same but with the 120 degrees phase difference. The asymmetry in lines and loads produces a certain level of unbalance in real power system and this is considered as disturbance whose level should be controlled to maintain the electromagnetic compatibility of the system [1].

For distribution system, in particular, the system is inherently unbalanced, due to factors such as the unbalance of customer loads, the presence of unsymmetrical line spacing, and the combination of single, double and three-phase line sections. Therefore, three-phase power flow is necessary to accurately simulate the unbalance system.

Inclusion of unbalance increases the dimension of problem

The Authors are with the Department of Electrical and Computer Engineering, Curtin University of Technology, Perth, WA, 6845, Australia, email: agus.ulinuha@postgrad.curtin.edu.au or guslin70@yahoo.com as all the three phases need to be considered instead of single phase balanced representation. On the other hand, distribution system commonly constructed as radial system with high $\mathrm{R} / \mathrm{X}$ ratio may cause the sophisticated power flow algorithms fail to converge. The robust algorithm for three-phase power flow is therefore needed.

Decomposition of the coupled unbalance system into positive, negative and zero symmetrical components is the most popular approach used in three-phase power flow algorithms [2, 3]. This eliminates the mutual coupling between phases so a three-phase power flow can be run three times, once for each phase. However, if the coupling between sequences occurs, then there is no real advantage in decomposing the system into the symmetrical components. Furthermore, this may result in significant error in calculation.

Another approach is decoupling the three-phase system into individual phases by introducing compensation current injections [4-7]. Therefore, a three-phase power flow can be solved independently for every phase without utilization of symmetrical components. All components are modeled by phase voltages, admittances and independent current sources. This approach will work well as long as every component can be modeled in the admittance matrix or can be converted into equivalent injection current.

The methods for three-phase distribution networks can be basically divided into two classes, Gauss-Seidel [4, 8] and Newton-Raphson [5, 9, 10]. The Gauss-Seidel method needs much iteration and is known to be slow. Newton-Raphson has good convergence characteristic, but the Jacobian that needs to be partially or totally calculated in every iteration makes this approach unattractive.

This paper presents three-phase power flow for unbalance distribution system using forward-backward propagation technique. The algorithm works directly on the system without any modification. Therefore, there is no need to decompose the system into symmetrical components as well as to decouple the system into individual phases. However, the conversion of load and shunt element into their equivalent injection current is necessary. Distribution line charging is usually too small to be included [5]. The algorithm is implemented on IEEE 34-bus system including asymmetrical lines and unbalance loads. 


\section{SYSTEM MODELING}

\section{A. Line Modeling}

The accuracy of three-phase power flow results greatly depends on the line impedance model used. Therefore, an exact model of a three-phase line section needs to be firstly developed. The model of distribution line feeder in [11] will be developed and used in this paper. An equivalent circuit for a three-phase line section is shown in Fig. 1.

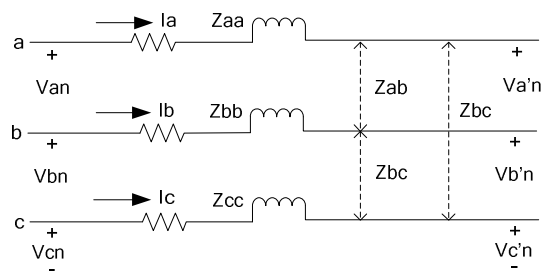

Fig. 1. Three-phase line model

The modeling of three-phase lines starts with determination of self and mutual impedances of a line section which are functions of the conductors and the spacing between conductors on the pole or underground. The "modified" form of Carson's equations are used to determine the self and mutual impedances of this model and are given by the following equations:

$z_{i i}=r_{i}+0.0953+j 0.12134 \times\left[\ln \left(1 / G M R_{i}\right)+7.934\right] \Omega / \mathrm{mi}$

$z_{i j}=0.0953+j 0.12134 \times\left[\ln \left(1 / D_{i j}\right)+7.934\right] \Omega / \mathrm{mi}$

Where $r_{i}$ is the conductor resistance $(\Omega /$ mile $), G M R_{i}$ is the conductor geometric mean radius $(\mathrm{ft})$, and $D_{i j}$ is the spacing between conductors $i$ and $j$ (ft). Application of (1) and (2) to the three-phase line indicated in Fig. 1 results in a $4 \times 4$ "primitive impedance matrix":

$[$ Zprim $]=\left[\begin{array}{cccc}z_{a a} & z_{a b} & z_{a c} & z_{a n} \\ z_{b a} & z_{b b} & z_{b c} & z_{b n} \\ z_{c a} & z_{c b} & z_{c c} & z_{c n} \\ z_{n a} & z_{n b} & z_{n c} & z_{n n}\end{array}\right]$

By applying Kron reduction, this matrix may be reduced into $3 \times 3$ "phase impedance matrix" whose elements are determined by the following equation:

$Z_{i j}=z_{i j}-z_{i n} z_{n j} / z_{n n}$

And the resulted "phase impedance matrix" is:

$\left[Z_{a b c}\right]=\left[\begin{array}{lll}Z_{a a} & Z_{a b} & Z_{a c} \\ Z_{b a} & Z_{b b} & Z_{b c} \\ Z_{c a} & Z_{c b} & Z_{c c}\end{array}\right]$

\section{B. Load Modeling}

The load (balance and unbalance) is represented by its equivalent injection current. The load modeling in [4] is adopted in this paper. For three-phase loads connected in wye or single-phase loads connected line to neutral, the equivalent injection currents at $k^{\text {th }}$ bus are determined by the following equation:
$I_{m}^{k}=\frac{P_{m}^{k}-j Q_{m}^{k}}{V_{m}^{k} *} ; m \in[a, b, c]:$ phase

Where $P_{m}, Q_{m}, V_{m}{ }^{*}$ denote real power, reactive power, and complex conjugate of the voltage phasor for each phase, respectively. For three-phase loads connected in delta or single-phase loads connected line to line, the equivalent injection currents at the $k^{\text {th }}$ bus are determined by the following equations:

$I_{a}^{k}=\frac{P_{a b}^{k}-j Q_{a b}^{k}}{V_{a}^{k} *-V_{b}^{k} *}-\frac{P_{c a}^{k}-j Q_{c a}^{k}}{V_{c}^{k} *-V_{a}^{k} *}$

$I_{b}^{k}=\frac{P_{b c}^{k}-j Q_{b c}^{k}}{V_{b}^{k} *-V_{c}^{k} *}-\frac{P_{a b}^{k}-j Q_{a b}^{k}}{V_{a}^{k} *-V_{b}^{k} *}$

$I_{c}^{k}=\frac{P_{c a}^{k}-j Q_{c a}^{k}}{V_{c}^{k} *-V_{a}^{k} *}-\frac{P_{b c}^{k}-j Q_{b c}^{k}}{V_{b}^{k} *-V_{c}^{k} *}$

Where $P_{n}, Q_{n}, n \in[a b, b c, c a]$, represents the real and reactive load connected between the respective phases, and $V_{m}{ }^{*}, m \in$ $[a, b, c]$ denotes the complex conjugate of the voltage phasor for each phase, respectively.

\section{Shunt Admittance Modeling}

Three phase shunt capacitors can also be represented by equivalent injection currents [4]. Assuming that the capacitor bank has an ungrounded wye connection, the current injection in each phase can be expressed by:

$I_{a}^{s h}=\frac{y^{s h}}{3}\left(-2 V_{a}^{k}+V_{b}^{k}+V_{c}^{k}\right)$

$I_{b}^{s h}=\frac{y^{s h}}{3}\left(V_{a}^{k}-2 V_{b}^{k}+V_{c}^{k}\right)$

$I_{c}^{s h}=\frac{y^{s h}}{3}\left(V_{a}^{k}+V_{b}^{k}-2 V_{c}^{k}\right)$

Where $y^{s h}=j Q^{0} \Lambda V^{0} I^{2} ; Q^{0}$ is the nominal reactive power per phase and $\left|V^{0}\right|$ is the magnitude of the nominal voltage each phase. If the capacitor bank has a grounded wye connection, then the injection current is:

$I_{a}^{s h}=-y^{s h} V_{a}^{k}$

$I_{b}^{s h}=-y^{s h} V_{b}^{k}$

$I_{c}^{s h}=-y^{s h} V_{c}^{k}$

\section{FORWARD-BACKWARD ALGORITHM}

Power flow is calculated by initially mapping the distribution network to determine forward and backward propagation paths followed by branch current calculation and bus voltages calculation.

\section{A. Branch Current Calculation}

Using backward propagation path, branch current can be calculated using the equivalent bus injection currents. The branch current is successively calculated for the network ends toward the source (swing). The voltage at each bus therefore 
needs to be firstly determined. For the first iteration, the voltage at each bus is set to $1.0 \mathrm{pu}$ with the angle of $0,-120$ and 120 degrees for phase $a, b$, and c, respectively. These voltages are updated during the iteration and therefore the injection currents will also change.

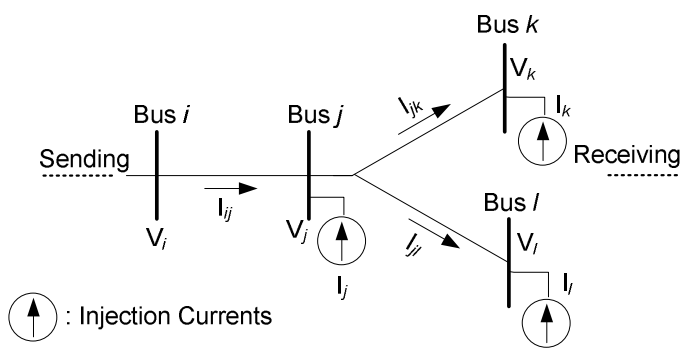

Fig. 2. Part of a distribution system

Fig. 2 indicates a part of distribution system and bus injection currents. The relationships between branch currents and injection currents are:

$I_{j k}=-I_{k}$

$I_{j l}=-I_{l}$

$I_{i j}=I_{j k}+I_{j l}-I_{k}$

Where $I_{j k}$ is the branch current between bus $j$ and bus $k$, and $I_{j}$ injection currents at bus, respectively.

\section{B. Bus Voltage Calculation}

Using forward propagation path, the voltage at each bus is calculated using the obtained branch currents and line impedance. The voltages are consecutively calculated from the source (swing) toward the network ends. The voltage of swing bus is kept constant at $1.0 \angle 0^{\circ} \mathrm{pu}, 1.0 \angle-120^{\circ} \mathrm{pu}$, and $1.0 \angle 120^{\circ}$ pu for phase $a, b$, and c, respectively. For the part of system indicated in Fig. 2, the bus voltages can be calculated as follows:

$V_{j}=V_{i}-Z_{i j} I_{i j}$

$V_{k}=V_{j}-Z_{j k} I_{j k}$

$V_{l}=V_{j}-Z_{j l} I_{j l}$

Where $V_{j}$ is the voltage at bus $j$ and $Z_{j k}$ is the impedance of line section between bus $j$ and $k$. The updated bus voltages are then used to calculate the bus injection currents. It should be noted that all calculations are carried in the three-phase frame.

\section{Convergence Criteria}

The outlined steps for branch currents and bus voltages calculations are invoked during the power flow iteration. The iteration converges if the different of bus voltages for the consecutive iterations is equal to or less than the prescribed tolerance. The voltage mismatch of bus $j$ at the $n^{\text {th }}$ iteration is given by:

$\Delta V_{j}^{n}=V_{j}^{n}-V_{j}^{n-1}$ for phase a, b, c

$\operatorname{Re}\left(\Delta V_{j}^{n}\right)<\varepsilon ; j \in$ all buses

$\operatorname{Im}\left(\Delta V_{j}^{n}\right)<\varepsilon ; j \in$ all buses
Where $\varepsilon$ is the prescribed tolerance. If these equations are satisfied, then the iteration stops. Otherwise, iteration process is repeated. Once the load flow iteration converges all the branch currents and voltage at each bus are known. The real and reactive power loss can therefore be calculated.

\section{Unbalance Analysis}

The system is unbalance if quantities of negative and zero sequence voltages can be observed in the system [1, 12]. Therefore, the level of unbalance may be measured using quantitative unbalance of voltage [13]. This can be calculated using the following equation:

$M V_{0}(j)=V_{0}(j) / V_{1}(j)$

$M V_{2}(j)=V_{2}(j) / V_{1}(j)$

Where $M V_{0}(j)$ and $M V_{2}(j)$ is the measurement of zero- and positive-sequence voltage with respect to the positive sequence voltage at bus $j$. The sequence component of voltage at bus $j$ can be calculated by:

$\left[\begin{array}{c}V_{0}(j) \\ V_{1}(j) \\ V_{2}(j)\end{array}\right]=1 / 3 *[T]\left[\begin{array}{l}V_{a}(j) \\ V_{b}(j) \\ V_{c}(j)\end{array}\right]$

Where $T$ is transformation matrix for symmetrical components. The three-phase power flow algorithm using ForwardBackward Propagation technique is given in the flowchart of Figure 3.

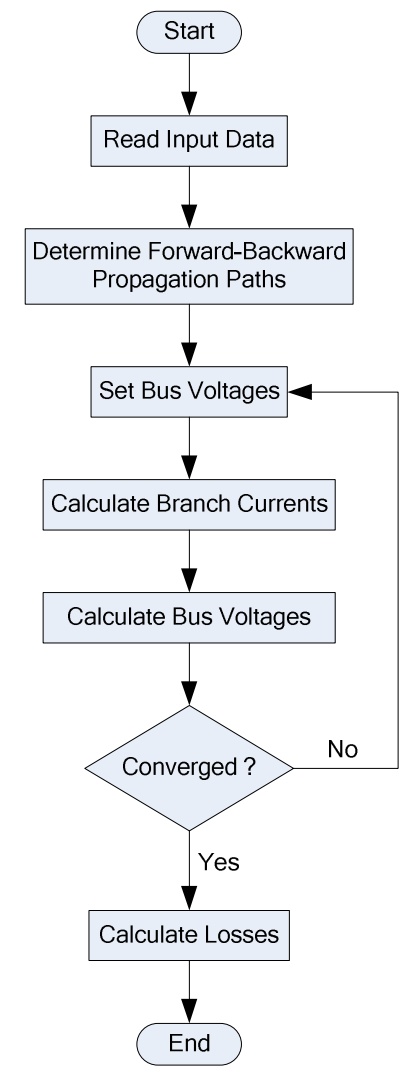

Fig. 3. The flowchart for three-phase power flow calculation using ForwardBackward Propagation technique 


\section{SimUlation}

\section{A. System Data}

The simulation is carried out for the IEEE 34-bus system [14] indicated in Fig. 4. The system includes balance as well as unbalance loads. A minor modification is performed for the system to only include three phase asymmetrical lines. However, the load data and the capacitor data are remain the same. The system load and branch data are respectively given in Table 1 (A and B) and Table 2.

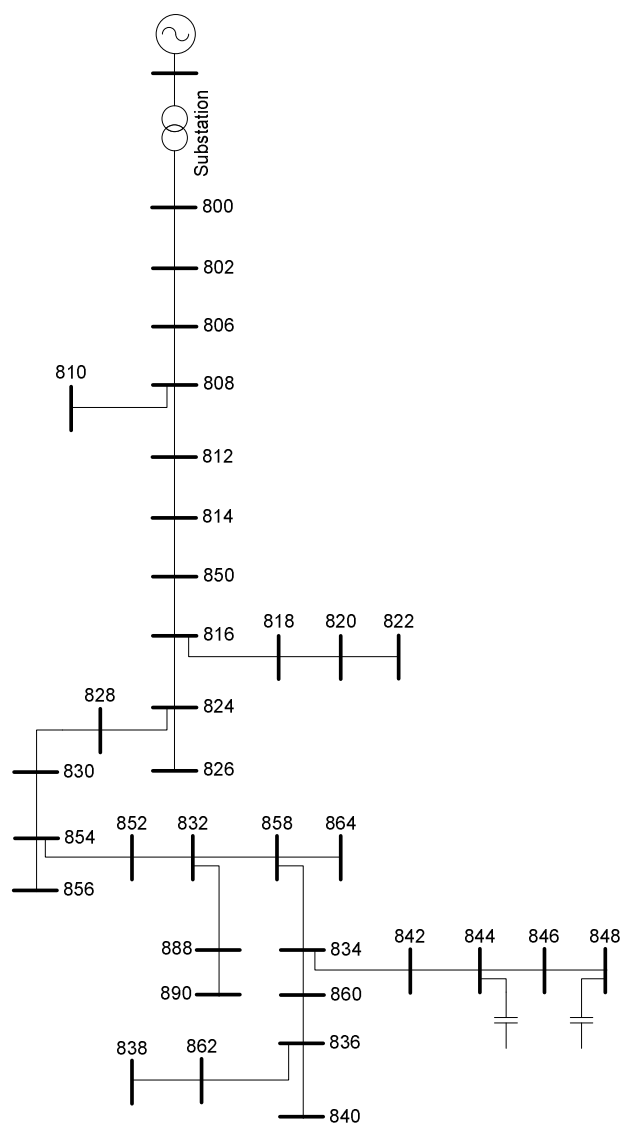

Fig. 4. The IEEE 34-bus system used for simulation

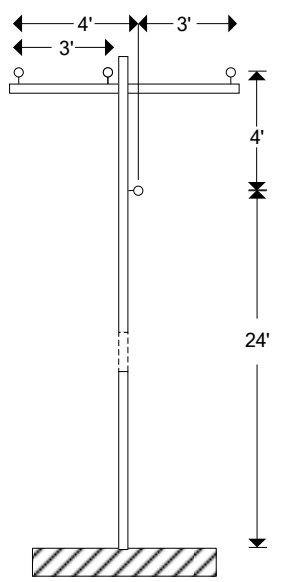

Fig. 5. Overhead line spacing for the IEEE 34-bus system
Fig. 5 shows the spacing distances between the phase conductors and the neutral conductor. The line configuration is given in Table 2 ( $3^{\text {rd }}$ column) indicating that the phase conductors are sequentially placed starting from the left side position.

TABLE I(A)

\begin{tabular}{|c|c|r|r|r|r|r|r|}
\hline \multicolumn{1}{|c|}{ BALANCE LOADS OF THE IEEE 34-BUS SYSTEM } \\
\hline Bus & $\mathrm{Y} /$ & \multicolumn{2}{|c|}{ Phase A } & \multicolumn{2}{|c|}{ Phase B } & \multicolumn{2}{|c|}{ Phase C } \\
\cline { 3 - 8 }$\#$ & $\Delta$ & $\mathrm{kW}$ & $\mathrm{kVAr}$ & \multicolumn{1}{|c|}{$\mathrm{kW}$} & $\mathrm{kVAr}$ & $\mathrm{kW}$ & $\mathrm{kVAr}$ \\
\hline 860 & $\mathrm{Y}$ & 19.91 & 15.94 & 19.91 & 15.94 & 19.91 & 15.94 \\
840 & $\mathrm{Y}$ & 8.86 & 7.09 & 8.86 & 7.09 & 8.86 & 7.09 \\
844 & $\Delta$ & 133.44 & 106.8 & 133.44 & 106.8 & 133.44 & 106.8 \\
848 & $\mathrm{Y}$ & 19.45 & 15.57 & 19.45 & 15.57 & 19.45 & 15.57 \\
890 & $\Delta$ & 27.00 & 21.62 & 27.00 & 21.62 & 27.00 & 21.62 \\
\hline
\end{tabular}

TABLE I(B)

UNBALANCE LOADS OF THE IEEE 34-BUS SYSTEM

\begin{tabular}{|c|c|r|r|r|r|r|r|}
\hline Bus & \multirow{2}{*}{ Y/ } & \multicolumn{2}{|c|}{ Phase A } & \multicolumn{2}{|c|}{ Phase B } & \multicolumn{2}{|c|}{ Phase C } \\
\cline { 3 - 8 } & & $\mathrm{kW}$ & $\mathrm{kVAr}$ & $\mathrm{kW}$ & $\mathrm{kVAr}$ & $\mathrm{kW}$ & $\mathrm{kVAr}$ \\
\hline 806 & $\mathrm{Y}$ & 0 & 0 & 31.2 & 16.1 & 26.07 & 13.8 \\
810 & $\Delta$ & 0 & 0 & 15.8 & 8.21 & 0 & 0 \\
820 & $\Delta$ & 33.9 & 17.5 & 0 & 0 & 0 & 0 \\
822 & $\mathrm{Y}$ & 135.5 & 70.0 & 0 & 0 & 0 & 0 \\
824 & $\mathrm{Y}$ & 0 & 0 & 0.39 & 0.2 & 0 & 0 \\
826 & $\mathrm{Y}$ & 0 & 0 & 41.9 & 21.6 & 0 & 0 \\
828 & $\Delta$ & 0 & 0 & 0 & 0 & 2.78 & 1.44 \\
830 & $\Delta$ & 6.18 & 3.2 & 0 & 0 & 0 & 0 \\
834 & $\mathrm{Y}$ & 3.99 & 2.06 & 12.5 & 6.49 & 12.82 & 6.63 \\
836 & $\Delta$ & 27.37 & 14.1 & 10.5 & 5.45 & 42.05 & 21.7 \\
838 & $\Delta$ & 27.61 & 14.2 & 0 & 0 & 0 & 0 \\
840 & $\mathrm{Y}$ & 17.49 & 9.04 & 21.8 & 11.2 & 0 & 0 \\
842 & $\mathrm{Y}$ & 0 & 0 & 0 & 0 & 0 & 0 \\
844 & $\Delta$ & 9.12 & 4.71 & 0 & 0 & 0 & 0 \\
846 & $\mathrm{Y}$ & 0 & 0 & 24.5 & 12.7 & 22.23 & 11.4 \\
848 & $\mathrm{Y}$ & 0 & 0 & 22.6 & 11.7 & 0 & 0 \\
856 & $\mathrm{Y}$ & 0 & 0 & 3.71 & 1.92 & 0 & 0 \\
858 & $\Delta$ & 6.68 & 3.45 & 1.08 & 0.56 & 5.35 & 2.77 \\
860 & $\mathrm{Y}$ & 15.66 & 8.09 & 20.8 & 10.7 & 111.1 & 57.4 \\
862 & $\mathrm{Y}$ & 0 & 0 & 0 & 0 & 0 & 0 \\
864 & $\Delta$ & 0.63 & 0.33 & 0 & 0 & 0 & 0 \\
\hline
\end{tabular}

Note

1. Swing: bus 800

2. MVA base: 2.5 MVA

TABLE II

BRANCH DATA OF THE IEEE 34-BUS SYSTEM

\begin{tabular}{|c|r|l|r|r|c|c|c|}
\hline \multicolumn{2}{|c|}{ Bus } & Config & length & \multicolumn{2}{|c|}{ Line Condctr } & \multicolumn{2}{c|}{ Neutral } \\
\cline { 1 - 5 } Fr & To & & (ft) & R & GMR & R & GMR \\
\hline 80 & 80 & BACN & 2580 & 1.6 & 0.0041 & 1.69 & 0.00418 \\
80 & 80 & BACN & 1730 & 1.6 & 0.0041 & 1.69 & 0.00418 \\
80 & 80 & BACN & 3223 & 1.6 & 0.0041 & 1.69 & 0.00418 \\
80 & 81 & BCAN & 5840 & 2.5 & 0.0045 & 2.55 & 0.00452 \\
80 & 81 & BACN & 3750 & 1.6 & 0.0041 & 1.69 & 0.00418 \\
81 & 81 & BACN & 2973 & 1.6 & 0.0041 & 1.69 & 0.00418 \\
81 & 85 & BACN & 10 & 1.6 & 0.0041 & 1.69 & 0.00418 \\
81 & 81 & ABCN & 1710 & 2.5 & 0.0045 & 2.55 & 0.00452 \\
81 & 82 & BACN & 1021 & 1.6 & 0.0041 & 1.69 & 0.00418 \\
81 & 82 & ABCN & 4815 & 2.5 & 0.0045 & 2.55 & 0.00452 \\
82 & 82 & ABCN & 1374 & 2.5 & 0.0045 & 2.55 & 0.00452 \\
82 & 82 & BCAN & 3030 & 2.5 & 0.0045 & 2.55 & 0.00452 \\
82 & 82 & BACN & 840 & 1.6 & 0.0041 & 1.69 & 0.00418 \\
82 & 83 & BACN & 2044 & 1.6 & 0.0041 & 1.69 & 0.00418 \\
83 & 85 & BACN & 520 & 1.6 & 0.0041 & 1.69 & 0.00418 \\
83 & 85 & BACN & 4900 & 1.6 & 0.0041 & 1.69 & 0.00418 \\
83 & 88 & BACN & 100 & 1.6 & 0.0041 & 1.69 & 0.00418 \\
83 & 86 & BACN & 2020 & 1.6 & 0.0041 & 1.69 & 0.00418 \\
83 & 84 & BACN & 280 & 1.6 & 0.0041 & 1.69 & 0.00418 \\
83 & 84 & BACN & 860 & 1.6 & 0.0041 & 1.69 & 0.00418 \\
\hline
\end{tabular}


TABLE II (CONTINUED)

BRANCH DATA OF THE IEEE 34-BUS SYSTEM

\begin{tabular}{|c|c|c|r|r|c|c|c|}
\hline \multicolumn{2}{|c|}{ Bus } & \multirow{2}{*}{ Config. } & \multicolumn{1}{c|}{ length } & \multicolumn{2}{c|}{ Line Condctr } & \multicolumn{2}{c|}{ Neutral Condctr } \\
\cline { 1 - 5 } Fr & To & & (ft) & R & GMR & R & GMR \\
\hline 836 & 862 & BACN & 280 & 1.69 & 0.00418 & 1.69 & 0.00418 \\
842 & 844 & BACN & 1350 & 1.69 & 0.00418 & 1.69 & 0.00418 \\
844 & 846 & BACN & 3640 & 1.69 & 0.00418 & 1.69 & 0.00418 \\
846 & 848 & BACN & 530 & 1.69 & 0.00418 & 1.69 & 0.00418 \\
850 & 816 & BACN & 310 & 1.69 & 0.00418 & 1.69 & 0.00418 \\
852 & 832 & BACN & 10 & 1.69 & 0.00418 & 1.69 & 0.00418 \\
854 & 856 & BCAN & 23330 & 2.55 & 0.00452 & 2.55 & 0.00452 \\
854 & 852 & BACN & 36830 & 1.69 & 0.00418 & 1.69 & 0.00418 \\
858 & 864 & ABCN & 1620 & 2.55 & 0.00452 & 2.55 & 0.00452 \\
858 & 834 & BACN & 5830 & 1.69 & 0.00418 & 1.69 & 0.00418 \\
860 & 836 & BACN & 2680 & 1.69 & 0.00418 & 1.69 & 0.00418 \\
862 & 838 & ACBN & 4860 & 1.69 & 0.00418 & 1.69 & 0.00418 \\
888 & 890 & BACN & 10560 & 1.12 & 0.00446 & 1.12 & 0.00446 \\
\hline
\end{tabular}

Note : $\mathrm{R}$ in $(\Omega / \mathrm{mi})$ and GMR in $(\mathrm{ft})$

\section{B. Simulation Results}

The system is run using the algorithm presented in Fig. 3. The simulation is coded using Matlab version 7.0 (R14). For the system of Fig.4, the three-phase power flow calculation takes 5 iterations to converge. This iteration number is fairly small indicating that the algorithm is robust for unbalance power flow calculation. The results of simulation including magnitude and angle of voltage at every phase are given in Table 3. The simulation also indicates the real and reactive power losses of $8.2 \mathrm{~kW}$ and $3.4 \mathrm{kVAr}$, respectively.

TABLE III

SimULATION RESUlts of THE IEEE 34-Bus UNBALANCE SySTEM

\begin{tabular}{|c|c|c|c|c|c|c|}
\hline bus & \multicolumn{2}{|c|}{ Volt at phase a } & \multicolumn{2}{c|}{ Volt at phase b } & \multicolumn{2}{c|}{ Volt at phase c } \\
\cline { 2 - 7 } no & mag & Angle & mag & Angle & mag & Angle \\
\hline 800 & 100 & 0.00 & 100 & -120.0 & 100 & 120.0 \\
802 & 99.9 & -0.01 & 99.9 & -120.0 & 99.9 & 119.9 \\
806 & 99.8 & -0.02 & 99.9 & -120.0 & 99.9 & 119.9 \\
808 & 99.0 & -0.22 & 99.2 & -120.2 & 99.1 & 119.8 \\
810 & 99.0 & -0.22 & 99.2 & -120.2 & 99.1 & 119.8 \\
812 & 97.9 & -0.45 & 98.4 & -120.4 & 98.3 & 119.5 \\
814 & 97.1 & -0.64 & 97.8 & -120.5 & 97.7 & 119.4 \\
816 & 97.1 & -0.64 & 97.7 & -120.5 & 97.6 & 119.4 \\
818 & 97.1 & -0.64 & 97.7 & -120.5 & 97.6 & 119.4 \\
820 & 96.3 & -0.66 & 97.8 & -120.5 & 97.6 & 119.4 \\
822 & 96.1 & -0.67 & 97.9 & -120.5 & 97.6 & 119.5 \\
824 & 97.0 & -0.69 & 97.5 & -120.5 & 97.4 & 119.3 \\
826 & 97.0 & -0.69 & 97.5 & -120.5 & 97.4 & 119.3 \\
828 & 97.0 & -0.69 & 97.5 & -120.5 & 97.4 & 119.3 \\
830 & 96.7 & -0.81 & 97.1 & -120.6 & 97.0 & 119.1 \\
832 & 96.2 & -1.02 & 96.4 & -120.8 & 96.1 & 118.8 \\
834 & 96.0 & -1.08 & 96.2 & -120.8 & 95.9 & 118.8 \\
836 & 96.0 & -1.08 & 96.2 & -120.8 & 95.9 & 118.8 \\
838 & 96.0 & -1.08 & 96.1 & -120.8 & 95.9 & 118.8 \\
840 & 96.0 & -1.08 & 96.2 & -120.8 & 95.9 & 118.8 \\
842 & 96.0 & -1.08 & 96.2 & -120.8 & 95.9 & 118.8 \\
844 & 96.0 & -1.09 & 96.2 & -120.9 & 95.9 & 118.7 \\
846 & 96.0 & -1.11 & 96.2 & -120.9 & 95.9 & 118.7 \\
848 & 96.0 & -1.11 & 96.2 & -120.9 & 95.9 & 118.7 \\
850 & 97.1 & -0.64 & 97.8 & -120.5 & 97.7 & 119.4 \\
852 & 96.2 & -1.02 & 96.4 & -120.8 & 96.1 & 118.8 \\
854 & 96.7 & -0.81 & 97.1 & -120.7 & 97.0 & 119.1 \\
856 & 96.7 & -0.81 & 97.1 & -120.6 & 97.0 & 119.1 \\
858 & 96.1 & -1.05 & 96.3 & -120.8 & 96.0 & 118.8 \\
860 & 96.0 & -1.08 & 96.2 & -120.8 & 95.9 & 118.8 \\
862 & 96.0 & -1.08 & 96.2 & -120.8 & 95.9 & 118.8 \\
864 & 96.1 & -1.05 & 96.3 & -120.8 & 96.0 & 118.8 \\
888 & 96.2 & -1.02 & 96.4 & -120.8 & 96.1 & 118.8 \\
890 & 96.1 & -1.02 & 96.3 & -120.8 & 96.1 & 118.8 \\
\hline
\end{tabular}

TABLE II

QUANTITATIVE VOLTAGE UNBALANCE OF THE IEEE 34-BUS UNBALANCE SYSTEM

\begin{tabular}{|c|c|c|}
\hline bus no & V0/V1(\%) & V2/V1 $(\%)$ \\
\hline 800 & 0 & 0 \\
802 & 0.0009 & 0.0037 \\
806 & 0.0015 & 0.0062 \\
808 & 0.0495 & 0.0730 \\
810 & 0.0495 & 0.0703 \\
812 & 0.1062 & 0.1652 \\
814 & 0.1519 & 0.2395 \\
816 & 0.1524 & 0.2403 \\
818 & 0.1641 & 0.2492 \\
820 & 0.5016 & 0.5007 \\
822 & 0.5989 & 0.5622 \\
824 & 0.1087 & 0.2293 \\
826 & 0.1039 & 0.2286 \\
828 & 0.1061 & 0.2286 \\
830 & 0.0670 & 0.2131 \\
832 & 0.1625 & 0.1871 \\
834 & 0.2026 & 0.1805 \\
836 & 0.2066 & 0.1830 \\
838 & 0.2066 & 0.1842 \\
840 & 0.2059 & 0.1831 \\
842 & 0.2027 & 0.1804 \\
844 & 0.2033 & 0.1799 \\
846 & 0.2052 & 0.1777 \\
848 & 0.2051 & 0.1776 \\
850 & 0.1519 & 0.2396 \\
852 & 0.1625 & 0.1871 \\
854 & 0.0670 & 0.2127 \\
856 & 0.0626 & 0.2120 \\
858 & 0.1806 & 0.1843 \\
860 & 0.2088 & 0.1805 \\
862 & 0.2066 & 0.1831 \\
864 & 0.1806 & 0.1844 \\
888 & 0.1625 & 0.1871 \\
890 & 0.1623 & 0.1870 \\
\hline
\end{tabular}

The analysis of system unbalance is carried out by measuring the quantitative unbalance of voltage. The ratio of the zero- and negative-sequence voltages with respect to the positive-sequence voltage is calculated using Equation (13) and related results are given in Table 2. It may be observed that the unbalance level of the simulated system is fairly low. This is indicated by small quantities of zero- and negativesequence voltages. Therefore, three-phase power flow enables measuring the unbalance level of the system studied. The unbalance assessment is useful for the purpose of maintaining the system balance level not to exceed the maximum allowable level.

\section{CONCLUSION}

Three-phase power flow for an unbalance distribution system is carried out using Forward-Backward Propagation Technique. The IEEE 34-bus unbalance system is used for simulation. The main conclusions are:

- The implemented algorithm works directly on the simulated system and, therefore, there is no need to transform the system into the symmetrical components as well as to decouple the three-phase into individual phases;

- The algorithm is robust for the radial unbalance distribution system with good convergence characteristic; 
- The unbalance level of three-phase system may only be assessed by performing three-phase power instead of singlephase (balance) power flow.

\section{REFERENCES}

[1] J. G. Mayordomo, M. Izzeddine, S. Martinez, R. Asensi, A. G. Exposito, and $\mathrm{W}$. Xu, "Compact and flexible three-phase power flow based on a full Newton formulation," IEE Proceedings-Generation, Transmission and Distribution, vol. 149, pp. 225-232, 2002.

[2] K. L. Lo and C. Zhang, "Decomposed three-phase power flow solution using the sequence component frame," IEE Proceedings-Generation, Transmission and Distribution, vol. 140, pp. 181-188, 1993.

[3] X. P. Zhang and H. Chen, "Asymmetrical three-phase load-flow study based on symmetrical component theory," IEE Proceedings-Generation, Transmission and Distribution, vol. 141, pp. 248-252, 1994.

[4] J. C. M. Vieira, Jr., W. Freitas, and A. Morelato, "Phase-decoupled method for three-phase power-flow analysis of unbalanced distribution systems," IEE Proceedings-Generation, Transmission and Distribution, vol. 151, pp. 568-574, 2004.

[5] W.-M. Lin and J.-H. Teng, "Three-phase distribution network fastdecoupled power flow solutions," International Journal of Electrical Power \& Energy Systems, vol. 22, pp. 375-380, 2000.

[6] C. S. Cheng and D. Shirmohammadi, "A three-phase power flow method for real-time distribution system analysis," IEEE Transactions on Power Systems, vol. 10, pp. 671-679, 1995.

[7] T. H. Chen, M. S. Chen, K. J. Hwang, P. Kotas, and E. A. Chebli, "Distribution system power flow analysis-a rigid approach," IEEE Transactions on Power Delivery vol. 6, pp. 1146-1152, 1991.

[8] J.-H. Teng, "A modified Gauss-Seidel algorithm of three-phase power flow analysis in distribution networks," International Journal of Electrical Power \& Energy Systems, vol. 24, pp. 97-102, 2002.

[9] P. A. N. Garcia, J. L. R. Pereira, S. Carneiro, Jr., V. M. da Costa, and N. Martins, "Three-phase power flow calculations using the current injection method," IEEE Transactions on Power Systems, vol. 15, pp. 508-514, 2000.

[10] V. M. da Costa, M. L. de Oliveira, and M. R. Guedes, "Developments in the analysis of unbalanced three-phase power flow solutions," International Journal of Electrical Power \& Energy Systems, vol. 29, pp. 175-182, 2007.

[11] W. H. Kersting and W. H. Phillips, "Distribution feeder line models," IEEE Transactions on Industry Applications, vol. 31, pp. 715-720, 1995.

[12] S. Segui-Chilet, F. J. Gimeno-Sales, S. Orts, G. Garcera, E. Figueres, M Alcaniz, and R. Masot, "Approach to unbalance power active compensation under linear load unbalances and fundamental voltage asymmetries," International Journal of Electrical Power \& Energy Systems, vol. 29, pp. 526-539, 2007.

[13] D. Thukaram, H. M. Wijekoon Banda, and J. Jerome, "A robust three phase power flow algorithm for radial distribution systems," Electric Power Systems Research, vol. 50, pp. 227-236, 1999.

[14] W. H. Kersting, "Radial distribution test feeders," IEEE Transactions on Power Systems, vol. 6, pp. 975-985, 1991. 